\title{
An LP based Fair Subcarrier Allocation Algorithm for Cooperative OFDMA Systems with Grouped Users
}

\author{
Anil Kumar \\ Department of E \& CE \\ NIT-Hamirpur (H.P.) India
}

\author{
Manoranjan Rai Bharti \\ Department of E \& CE \\ NIT-Hamirpur (H.P.) India
}

\begin{abstract}
Cooperative communication and orthogonal frequency division multiplexing (OFDM) technology are both promising candidates for next generation wireless communication systems. In this paper a Linear Programming (LP) based subcarrier allocation algorithm for cooperative multiuser OFDM system with grouped user is proposed. The proposed algorithm maximizes the data rate of all users over downlink under given constraints over non-sharing of carriers and fairness of data rate among all users. In this proposed algorithm the non sharing constraint of subcarriers have been relaxed initially into sharing constraint so that the non-convex optimization problem becomes a convex optimization problem, and thus, can solved efficiently using standard convex optimization tools. After maximizing total capacity of all active users in the system, the subcarrier allocation constraint is, then converted back to non-sharing constraint. The performance of proposed algorithm is studied and simulation results show that higher total capacity can be achieved using the proposed algorithm.
\end{abstract}

\section{Keywords}

Cooperative wireless network, subcarrier allocation, orthogonal frequency division multiple access, fairness

\section{INTRODUCTION}

In wireless communication systems, the demand for higher and higher transmission rate is ever increasing. Orthogonal Frequency Division Multiple Access (OFDMA) is an effective technique for combating channel noise, multipath effects and enabling high data rate transmissions [1] over fading channels. Meanwhile, cooperative communication has become very promising in various wireless systems [2] because of its attractive features such as high throughput, low power consumption and wide cell coverage. Therefore, the incorporation of OFDMA and cooperative communication can provide a promising structure that offers more reliable services for the next generation wireless networks. In cooperative communication, the users can be grouped into a number of groups and partners of a group cooperate one another for their communication. A cooperative system thus may be consider as a virtual antenna array, where each antenna in the array corresponds to one of the partners. The partners can overhear each other's transmissions though the wireless medium, process this information and re-transmit to collaborate. This provides diversity of the transmitter signals at the corresponding receivers, and hence, can improve the transmission and reception in wireless communication system. Radio resource allocation is an important task in OFDMA based cooperative communication systems, wherein, users in the system are allocated resources, such as subcarrier, power etc, optimally. Many researchers have been working in this area of resource allocation in cooperative OFDMA systems. Jang and Lee proposed the rate maximization problem [3] and proved that the sum capacity is maximized when each subchannel is assigned to the user with the best subchannel gain and power is distributed by the water-filling algorithm. However, fairness is not considered in [3]. When the path loss differences among users are large, it is possible that the users with higher average channel gains will get allocated most of the resources, i.e., subchannels and power, for a significant portion of time. The users with lower average channel gains may not be able to receive any data, since most of the time the subchannels will assigned to users with higher channel gains. Resource Allocation problems are in general $\mathrm{NP}-\mathrm{Hard}$ [4]-[5] and can rapidly becomes intractable as the number of users and subcarriers increases. However, problem can be solved if we resort to a relaxed problem formulation wherein a non-convex optimization problem is converted to a convex optimization problem, and thus, can be solved efficiently using standard convex optimization tools e.g. "linprog".

The main objective of this research work is to devise efficient subcarrier allocation for grouped cooperative users in OFDMA systems. A cooperative wireless network downlink system on a TDMA based relaying protocol with multiple cooperative pairs of users who can relay their respective partner's messages are considered. It is assumed that users are employing Amplify and Forward (AF) relaying scheme [6], where each user forwards an amplified version of the signals, received from Base Station (BS), to its partner in the cooperation phase. This approach is one of the most widely adopted schemes in the literature due to its lower cost, simpler design and ability to achieve full diversity through simple operations compared to Decode and Forward (DF) relays. In this paper, an LP based subcarrier allocation algorithm for cooperative OFDMA system with grouped user is proposed.

The remainder of this paper is organized as follows. In section 2 , system model is introduced along with appropriate assumptions. Section 3 describes problem formulation. In section 4, an LP based fair subcarrier allocation algorithm is proposed. In section 5, simulation results have been discussed. Finally, the paper is concluded in section 6 .

\section{SYSTEM MODEL AND ASSUMPTIONS}

The cooperative OFDMA system model is shown in Fig. 1. There are $\mathrm{G}$ groups $(1 \leq \mathrm{g} \leq \mathrm{G})$ and in each group number of users is $\mathrm{U}(1 \leq \mathrm{u} \leq \mathrm{U})$. So total number of users in system is $\mathrm{G} \times \mathrm{U}$. Total $\mathrm{N}$ subcarriers $(1 \leq \mathrm{n} \leq \mathrm{N})$ is considered. Total number of users in each group is two i.e. $\mathrm{U}=2$ is considered. A downlink scenario is considered and for simplicity of analysis, a flat power allocation is assumed. The total transmit power at the base station is assumed to be $\mathrm{P}$ over the whole bandwidth; therefore, transmit power over each subcarrier is $\mathrm{P} / \mathrm{N}$. Let total bandwidth of system is B Hz. Therefore, each subcarrier is occupying a bandwidth of $(\mathrm{B} / \mathrm{N}) \mathrm{Hz}$. The 
wireless channel is modeled as frequency selective Rayleigh fading channel. Assume $(\mathrm{B} / \mathrm{N})$ is far less than the coherence bandwidth of channel, thus the channel response over each subcarrier is flat. Consider Additive White Gaussian Noise (AWGN) is present with single sided noise power spectral density (PSD) of $\mathrm{N}_{0}$ for all subcarriers and all users.

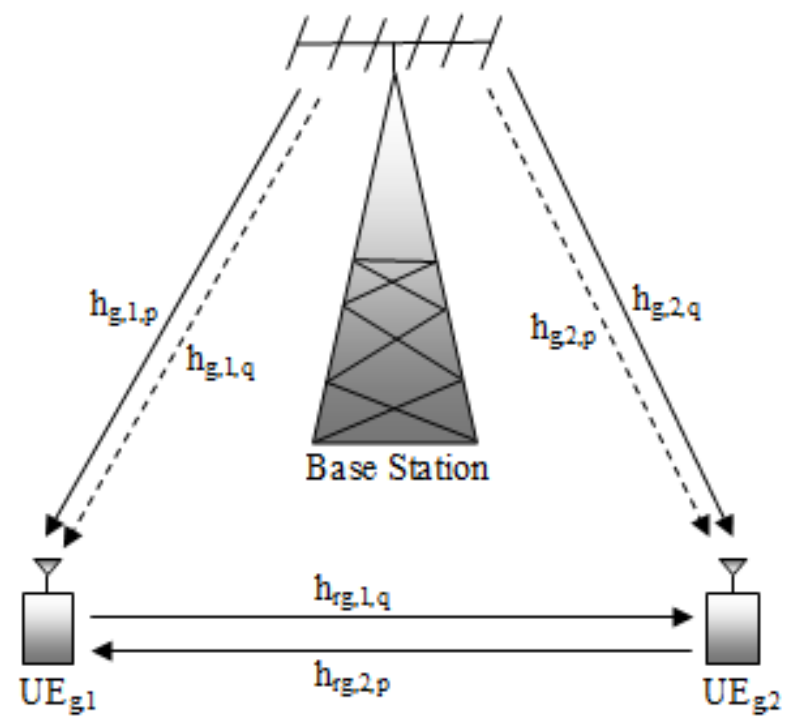

Fig. 1. System Model, $\mathrm{U}=2$

For simplicity of analysis, a TDMA based Amplify and Forward (AF) cooperation protocol is considered with twophases of operation: broadcast and cooperation. Broadcast of data by Base Station occurs in first time slot while the cooperation for transmission of data among users occurs in second and third time slots. In broadcast phase (the first timeslot), base station transmits the data to various users (in this case grouped users are $\mathrm{UE}_{\mathrm{g}, 1}$ and $\mathrm{UE}_{\mathrm{g}, 2}$ ). In cooperation phase (second and third timeslot), $\mathrm{UE}_{\mathrm{g}, 1}$ and $\mathrm{UE}_{\mathrm{g}, 2}$ after receiving the other user's signal in the first timeslot, amplify and forward it in the second and third timeslot respectively. It is assumed that each subcarrier is assigned to one and only one user i.e., none of the users are sharing subcarriers. According to Fig. 1, the $\mathrm{p}^{\text {th }}$ subcarrier is allocated to $\mathrm{UE}_{\mathrm{g}, 1}$ and the $\mathrm{q}^{\text {th }}$ subcarrier to $\mathrm{UE}_{\mathrm{g}, 2}$. Let us denote the channel gain on the $\mathrm{p}^{\text {th }}$ and $\mathrm{q}^{\text {th }}$ subcarrier between BS and $\mathrm{UE}_{\mathrm{g}, 1}$ as $\mathrm{h}_{\mathrm{g}, 1, \mathrm{p}}$ and $\mathrm{h}_{\mathrm{g}, 1, \mathrm{q}}$ respectively and between BS and user $\mathrm{UE}_{\mathrm{g}, 2}$ as $\mathrm{h}_{\mathrm{g}, 2, \mathrm{p}}$ and $h_{\mathrm{g}, 2, \mathrm{q}}$ respectively. Here $\mathrm{h}_{\mathrm{rg}, 1, \mathrm{q}}$ and $\mathrm{h}_{\mathrm{rg}, 2, \mathrm{p}}$ are the inter user channel gains from $\mathrm{UE}_{\mathrm{g}, 1}$ to $\mathrm{UE}_{\mathrm{g}, 2}$ and $\mathrm{UE}_{\mathrm{g}, 2}$ to $\mathrm{UE}_{\mathrm{g}, 1}$ respectively. A maximal ratio combiner is employed at each grouped user $\left(\mathrm{UE}_{\mathrm{g}, 1}\right.$ and $\left.\mathrm{UE}_{\mathrm{g}, 2}\right)$ to combine the received signal from the direct and indirect paths.

Let $d_{g, u}$ is the data stream of $\mathrm{UE}_{\mathrm{g}, \mathrm{u}}$ of $\mathrm{g}^{\text {th }}$ group. The signal received at the $\mathrm{UE}_{\mathrm{g}, 1}$ and $\mathrm{UE}_{\mathrm{g}, 2}$ during the first time slot is given by [7]:

$$
\begin{aligned}
& y_{g, 1, p}=\sqrt{\frac{P T}{N}} h_{g, 1, p} d_{g, 1}+n_{g, 1, p} \\
& y_{g, 1, q}=\sqrt{\frac{P T}{N}} h_{g, 1, q} d_{g, 2}+n_{g, 1, q} \\
& y_{g, 2, q}=\sqrt{\frac{P T}{N}} h_{g, 1, q} d_{g, 2}+n_{g, 1, q}
\end{aligned}
$$

$$
y_{g, 2, p}=\sqrt{\frac{P T}{N}} h_{g, 2, p} d_{g, 1}+n_{g, 2, p}
$$

Where $y_{g, u, p}$ is the received signal at $\mathrm{UE}_{\mathrm{g}, \mathrm{u}}(\mathrm{u}=1,2)$ on the $\mathrm{p}^{\text {th }}$ subcarrier. $\mathrm{n}_{\mathrm{g}, \mathrm{u}, \mathrm{p}}$ is AWGN added to the received signal at $\mathrm{UE}_{\mathrm{g}, \mathrm{u}}$ on the $\mathrm{p}^{\mathrm{th}}$ subcarrier. $\left(\frac{P T}{N}\right)$ is the transmit energy of $\mathrm{UE}_{\mathrm{g}, \mathrm{u}}$ over one symbol period, $\mathrm{T}$.

Now Each user $\mathrm{UE}_{\mathrm{g}, \mathrm{u}}$ normalizes the received signal by a factor of $\sqrt{\varepsilon\left\{\left|y_{g, u, p}\right|^{2}\right\}}$ (so that the average energy is unity) and retransmits it to other user (its partner) in the second or third timeslot with the same energy of $\left(\frac{P T}{N}\right)$. The received signal, $y_{r g, u, p}$ in the second and third timeslots at $\mathrm{UE}_{\mathrm{g}, \mathrm{u}}$ is given as [7]:

$$
\begin{aligned}
& y_{r g, 1, p}=\sqrt{\frac{P T}{N}} h_{r g, 2, p} \frac{y_{g, 2, p}}{\sqrt{\varepsilon\left\{\left|y_{g, 2, p}\right|^{2}\right\}}}+n_{r g, 1, p} \\
& y_{r g, 2, q}=\sqrt{\frac{P T}{N}} h_{r g, 1, q} \frac{y_{g, 1, q}}{\sqrt{\varepsilon\left\{\left|y_{g, 1, q}\right|^{2}\right\}}}+n_{r g, 2, q}
\end{aligned}
$$

Where $n_{r g, u, p}$ is the additive noise at $\mathrm{UE}_{\mathrm{g}, \mathrm{u}}$ on the $\mathrm{p}^{\text {th }}$ subcarrier and $\varepsilon\left\{\left|y_{g, u, p}\right|^{2}\right\}$ is the average energy of the received signal at $\mathrm{UE}_{\mathrm{g}, \mathrm{u}}$ in the first timeslot. The maximum error-free data rate of $\mathrm{UE}_{\mathrm{g}, \mathrm{u}}(\mathrm{u}=1,2)$ can be written as follows [8]:

$$
\begin{aligned}
& r_{g, 1, p}=\frac{B}{3 N} \log _{2}\left\{1+\frac{P}{N_{0} B}\left(\left|h_{g, 1, p}\right|^{2}+\psi_{g, 2, p}\left|h_{g, 2, p}\right|^{2}\right)\right\} \\
& r_{g, 2, q}=\frac{B}{3 N} \log _{2}\left\{1+\frac{P}{N_{0} B}\left(\left|h_{g, 2, q}\right|^{2}+\psi_{g, 1, q}\left|h_{g, 1, q}\right|^{2}\right)\right\}
\end{aligned}
$$

Where $\psi_{g, u, p}$ is a cooperation coefficient parameter and represent the contribution level from $\mathrm{UE}_{\mathrm{g}, \mathrm{u}}$ for the data rate of the user to which the $\mathrm{p}^{\text {th }}$ subcarrier is allocated. $\psi_{g, 1, q}$ and $\psi_{g, 2, p}$ are given as:

$$
\begin{aligned}
\psi_{g, 1, q} & =\frac{\left|h_{r g, 1, q}\right|^{2}}{1+\frac{P}{N_{0} B}+\left|h_{r g, 1, q}\right|^{2}} \\
\psi_{g, 2, p} & =\frac{\left|h_{r g, 2, p}\right|^{2}}{1+\frac{P}{N_{0} B}+\left|h_{r g, 2, p}\right|^{2}}
\end{aligned}
$$

It should be noted that the cooperation coefficient $\psi_{g, u, p}$ in an $\mathrm{AF}$ protocol cannot exceed one, which means that the impact of cooperation in the capacity from the indirect path is always less than the direct path.

\section{PROBLEM FORMULATION}

In this section, subcarrier allocation problem is formulated to determine the optimal assignment of subcarriers that maximizes the total capacity from all the sources to the destination. A cooperative multiuser OFDM system with $\mathrm{N}$ subcarriers and $\mathrm{G}$ groups of $\mathrm{U}$ cooperative users has been considered. Let $\rho_{\mathrm{g}, \mathrm{u}, \mathrm{n}}$ be a binary decision variable representing the assignment of subcarrier to user from a group. When $\rho_{\mathrm{g}, \mathrm{u}, \mathrm{n}}=1$, this means that subcarrier $\mathrm{n}$ is assigned to user $\mathrm{u}$ in a group $\mathrm{g}$ and when $\rho_{\mathrm{g}, \mathrm{u}, \mathrm{n}}=0$, this means otherwise. None of the users shares subcarrier, therefore in case $\rho_{\mathrm{g}, \mathrm{u}, \mathrm{n}}=1$ then $\rho_{\mathrm{g}^{\prime}, \mathrm{u}^{\prime}, \mathrm{n}}=0$ for all $\mathrm{g}^{\prime} \neq \mathrm{g}$ and $\mathrm{u}^{\prime} \neq \mathrm{u}$.

After subcarrier allocation, the total data rate of the two users in the $\mathrm{g}^{\text {th }}$ group can be written as: 


$$
\begin{aligned}
R_{g, 1} & =\sum_{n=1}^{N} \rho_{g, 1, n} r_{g, 1, n} \\
R_{g, 2} & =\sum_{n=1}^{N} \rho_{g, 2, n} r_{g, 2, n}
\end{aligned}
$$

Based on fairness consideration and flat power allocation, the optimization problem in a cooperative network with the objective to maximize the downlink data rate of the system can be formulated as:

$$
\max _{\rho_{g, u, n}} \sum_{g=1}^{G}\left(R_{g, 1}+R_{g, 2}\right)
$$

Subject to:

$$
\begin{array}{ll}
\mathrm{C} 1: & \rho_{g, u, n} \in\{0,1\}, \forall g, u, n \\
\mathrm{C} 2: & \Sigma_{g=1}^{G} \Sigma_{u=1}^{2} \rho_{g, u, n}=1, \quad \forall n \\
\mathrm{C} 3: & R_{1,1}=R_{1,2}=\ldots=R_{G, 1}=R_{G, 2}
\end{array}
$$

Constraints $\mathrm{C} 1$ and $\mathrm{C} 2$ state that subcarrier sharing is not allowed and constraint $\mathrm{C} 3$ reflecting the fairness consideration i.e. equal data rate requirement for all users.

\section{LINEAR PROGRAMMING BASED ALGORITHM}

In this section, a novel algorithm based on Linear programming technique is proposed. The optimization problem stated in (11) is an integer linear programming (ILP) problem. One challenging aspect of this problem in the context of OFDMA is the discrete nature of subcarrier assignment, which is the hard problem to solve. Therefore, finding the optimal solution for this non-convex problem requires searching through all the possible user and subcarrier allocations, which is prohibitively complex to employ in large system. However, to make the problem tractable, the integer constraints on $\rho_{\mathrm{g}, \mathrm{u}, \mathrm{n}}$ is relaxed by allowing time sharing of each subcarrier, which means that $\rho_{\mathrm{g}, \mathrm{u}, \mathrm{n}}$ can take on any value between 0 and 1 . The duality gap of any optimization problem satisfying the time-sharing condition is negligible as the number of subcarriers becomes sufficiently large [9]. Once optimization problem is relaxed by allowing time-sharing of each subcarrier, it can be solved by using the dual method and the solution is optimal [9].

The basic idea in this proposed algorithm is that once the subcarrier time sharing solution is available for this problem, then each subcarrier can be enforced to non-time sharing by allocating the subcarriers to the user whose sharing for the subcarrier is the most.

Now relaxed linear optimization problem can be written as:

$$
\max _{\rho_{g, u, n}} \sum_{g=1}^{G}\left(R_{g, 1}+R_{g, 2}\right)
$$

Subject to:

$$
\begin{array}{ll}
\mathrm{C} 1: & 0 \leq \rho_{g, u, n} \leq 1, \forall g, u, n \\
\mathrm{C} 2: & \Sigma_{g=1}^{G} \Sigma_{u=1}^{2} \rho_{g, u, n}=1, \quad \forall n \\
\mathrm{C} 3: & R_{1,1}=R_{1,2}=\ldots=R_{G, 1}=R_{G, 2}
\end{array}
$$

In this problem constrint $\mathrm{C} 1$ indicates the time sharing of subcarriers. Satisfying constraint $\mathrm{C} 1$ and $\mathrm{C} 2$ simultaneously suggest that the condition $\rho_{g, u, n} \leq 1$ on constraint $\mathrm{C} 1$ can be dropped as it is redundant. Therefore, final optimization problem becomes:

$$
\max _{\rho_{g, u, n}} \sum_{g=1}^{G}\left(R_{g, 1}+R_{g, 2}\right)
$$

Subject to:

$$
\begin{array}{ll}
\mathrm{C} 1: & \rho_{g, u, n} \geq 0, \forall g, u, n \\
\mathrm{C} 2: & \Sigma_{g=1}^{G} \Sigma_{u=1}^{2} \rho_{g, u, n}=1, \quad \forall n \\
\mathrm{C} 3: & R_{1,1}=R_{1,2}=\cdots=R_{G, 1}=R_{G, 2}
\end{array}
$$

\subsection{Use of "linprog" to get the optimal solution}

Now (13) can be solved by using any existing LP solver, which can be based on primal-dual method. One such existing function in MATLAB simulation tool is "linprog" which is based on Linear Interior Point Solver [10] and it is a variant of Mehrotra's predictor-corrector algorithm [11], a primal-dual interior-point method. A number of preprocessing steps need to be carried out before the algorithm begins to iterate. The standard form of the LP that can be solved by "linprog" is

$$
\min _{x} f^{T} x
$$

Subject to:

$$
\begin{aligned}
& \text { A. } x \leq b \\
& \text { Aeq. } x=\text { beq }
\end{aligned}
$$

Where $f, x, b$ and $b e q$ are vectors and $A$ and $A e q$ are matrices. Here $x$ is the list of variables, $f$ is the list of coefficient of variables in $x$ for the objective function. Now there are two type of constraints in the standard form of LP. One where all the constraints are in the form of $A . x \leq b$ and other where all the constraints are in the form of Aeq. $x=$ beq. All the coefficient of variables in the first kind of constraint will form the Matrix $A$ and all the constants in the first kind of constraint will form the Vector $b$. Similarly all the coefficients of variables in the second kind of constraint will form the Matrix Aeq and all the constants in the second kind of constraint will form the Vector beq.

\subsection{Mapping of optimization problem into standard form}

In this section, the algorithm to map the the optimization problem into standard form is presented. This mapping is required so that the standard "linprog" function can be used directly in the MATLAB simulations. This has the form as below:

$$
x=\operatorname{linprog}(f, A, b, \text { Aeq, beq })
$$

Where $x$ represents the solution vector. In the optimization problem under consideration this $x$ represents the subcarrier allocations to different users in the system. The generation of different vectors and matrices required to obtain the solution vector $x$ is explained in the steps given below:

\section{Step 1: Creating vector $f$}

In this step, equation (15) is used to create vector $f$.

$$
\sum_{g=1}^{G}\left(R_{g, 1}+R_{g, 2}\right)=\sum_{g=1}^{G} \sum_{u=1}^{2} \sum_{n=1}^{N}\left(\rho_{g, u, n} \times r_{g, u, n}\right)
$$


Note that standard objective function is for minimization, but objective function given in (13) is for maximization. Therefore, in order to make use of standard form, the element of vector $f$ need to be negated. The following algorithm describes the creation of vector $f$ :

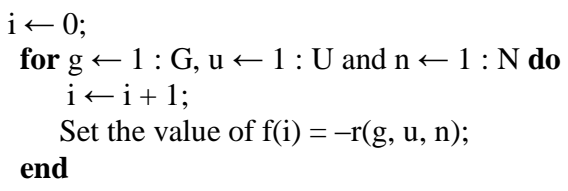

\section{Step 2: Creating matrix $A$ and vector $b$}

Matrix A contains all the inequality constraints and corresponding constants will form vector b. In the optimization problem, the only inequality constraint is $\mathrm{C} 1$, as given in (13), i.e., $\rho_{g, u, n} \geq 0, \forall g, u, n$. Now, because the standard form of inequality constraint is $A . x \leq b$, therefore, the values of constraint $\mathrm{C} 1$ need to be negated. Thus constraint $\mathrm{C} 1$ can be written as $-\rho_{g, u, n} \leq 0, \forall g, u, n$. Vector $\mathrm{b}$ will contain all the zeros. The following algorithm illustrates the generation of Matrix A and vector $b$.

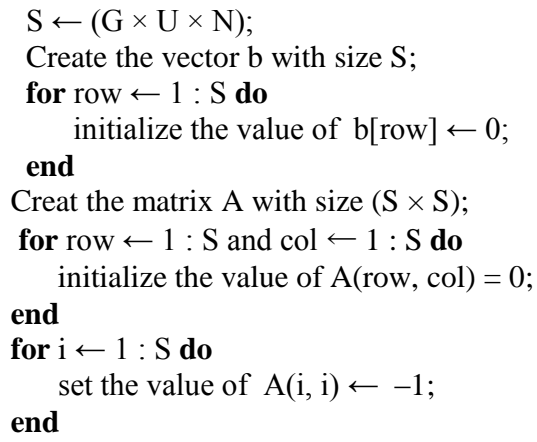

\section{Step 3: Creating column vector beq}

In order to generate matrix Aeq and vector beq, constraints $\mathrm{C} 2$ and $\mathrm{C} 3$, given in (13), will be used together. In this step the generation of column vector beq is discussed. As seen from constraints $\mathrm{C} 2$ and $\mathrm{C} 3$, the total number of elements in column vector beq will be $[N+(G \times U)-1]$. Due to constraint $\mathrm{C} 2$, the first $\mathrm{N}$ elements of column vector beq will be filled with the constant value of unity. The remaining [ $(\mathrm{G}$ $\times U)-1$ ] elements of column vector beq, are assigned zero value from constraint $\mathrm{C} 3$. The corresponding algorithm to generate vector beq is given as:

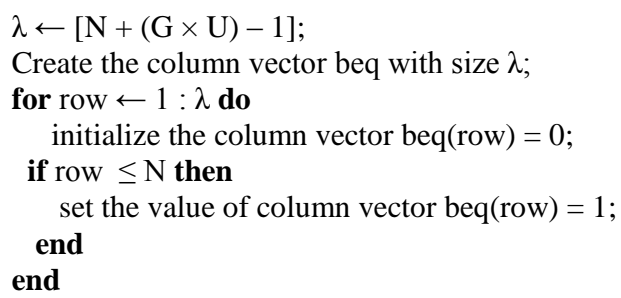

\section{Step 4: Creating matrix Aeq}

The steps 4 to 8 explain the generation of matrix Aeq, having $[\mathrm{N}+(\mathrm{G} \times \mathrm{U})-1]$ rows and $[\mathrm{G} \times \mathrm{U} \times \mathrm{N}]$ columns. In this particular step 4, matrix Aeq is initialized as below:

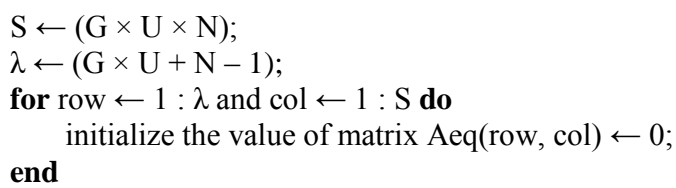

\section{Step 5: Assigning Aeq element values based on} constraint $C 2$

As per constraint $\mathrm{C} 2$, given in (13), the summation of shared part of each of subcarriers must be equal to unity. The following algorithm explains how constraint $\mathrm{C} 2$ can be mapped onto matrix Aeq to form its elements in first $\mathrm{N}$ rows.

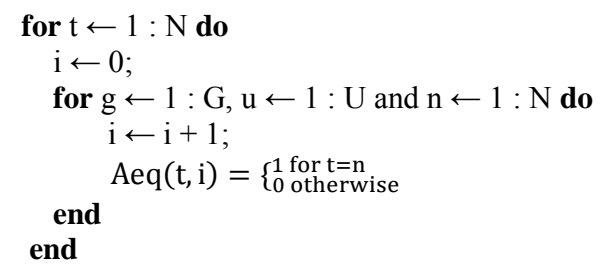

Step 6: Assigning Aeq elements values based on constraint C3-"Data rate of each first user in a group is equal"

According to constraint $\mathrm{C} 3$, in equation (13), the total data rate for each user in the system is equal. In this step, the following equality of data rates of first user in each group is mapped on to matrix Aeq:

$\mathrm{R}_{1,1}=\mathrm{R}_{2,1}=\ldots \ldots . .=\mathrm{R}_{\mathrm{G}, 1}$. This can be written in different $(\mathrm{G}-1)$ equations.

$$
\mathrm{R}_{1,1}-\mathrm{R}_{2,1}=0 ; \mathrm{R}_{2,1}-\mathrm{R}_{3,1}=0 ; \ldots \ldots . . \mathrm{R}_{\mathrm{G}-1,1}-\mathrm{R}_{\mathrm{G}, 1}=0 ;
$$

This can be explained in the following algorithm:

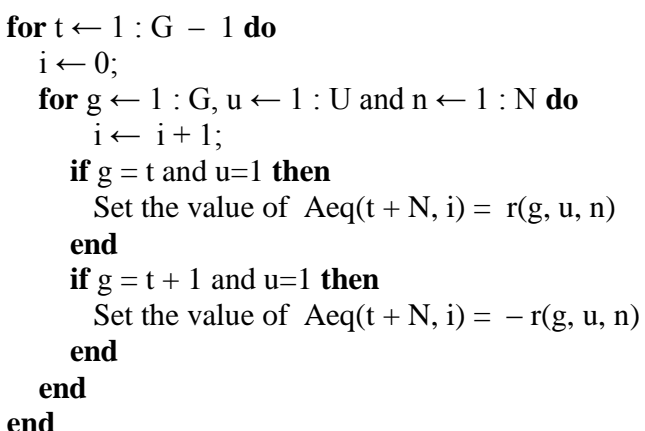

Step 7: Assigning Aeq elements values based on constraint C3-"Data rate of first and second user is equal"

Using constraint $\mathrm{C} 3$ again, the following equality of data rates is mapped on to matrix Aeq as follows:

$R_{1,1}=R_{1,2}$ i.e. $R_{1,1}-R_{1,2}=0$. The corresponding algorithm is as:

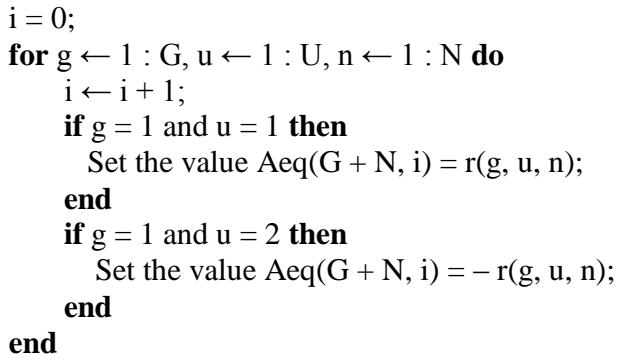

Step 8: Assigning Aeq elements values based on constraint C3-"Data rate of each second user in a group is equal"

The remaining elements of matrix Aeq will be assigned values based on the following equality of data rates. 
$\mathrm{R}_{1,2}=\mathrm{R}_{2,2}=\ldots \ldots=\mathrm{R}_{\mathrm{G}, 2}$.

i.e.

$\mathrm{R}_{1,2}-\mathrm{R}_{2,2}=0 ; \mathrm{R}_{2,2}-\mathrm{R}_{3,2}=0 ; \ldots \ldots \ldots ; \mathrm{R}_{\mathrm{G}-1,2}-\mathrm{R}_{\mathrm{G}, 2}=0$

The corresponding algorithm can be written as:

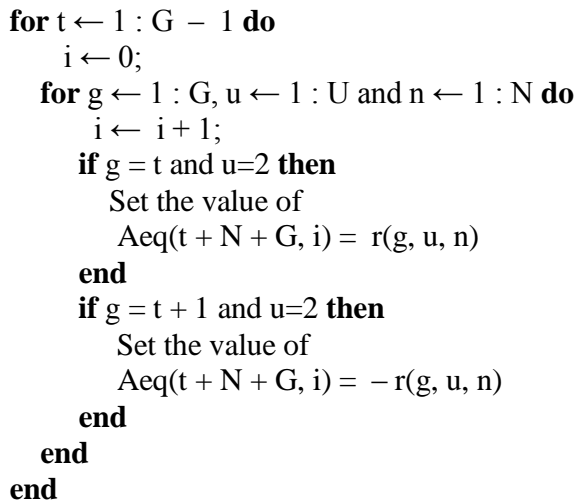

\subsection{Final subcarrier allocation}

After generating the required matrices and vectors the "linprog" function in MATLAB, is now used to get a solution vector $x$. This solution vector, $x$, actually represents the subcarrier assignment $\rho_{g, u, n}$, in given optimization problem. The mapping of solution vector $x$ into subcarrier assignment indicator $\rho_{g, u, n}$ can be described as:

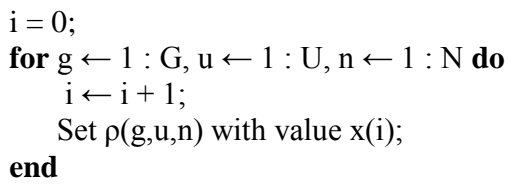

It is seen in (13) that $\rho_{g, u, n}$ takes on real values between 0 and 1. This means that solution thus obtained for subcarrier assignment is a time sharing solution. Optimization objective in (11), however, is to allocate subcarriers on non-sharing basis to different user in the system. In order to convert this time sharing solution to a non-time sharing solution, each subcarrier is allocated to only a single user whose sharing for that subcarrier is the most. Following algorithm describes this:

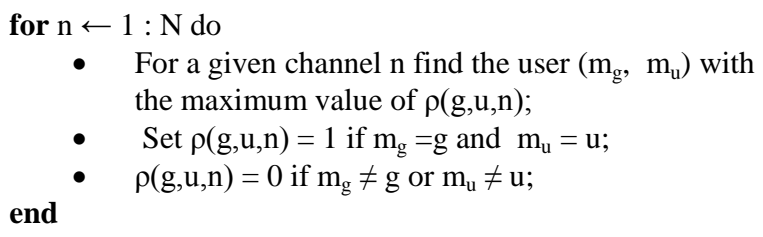

\subsection{Fairness}

It is very common to define the fairness in terms of data rates, where the objective is to allocate the resources to the users such that the users achieve the same data rate. Assuming equal data rate requirements among the users, the fairness index, $F_{p}$ is defined as [8]:

$$
F_{p}=\frac{\left(\Sigma_{g=1}^{G} \Sigma_{u=1}^{2} R_{g, u}\right)^{2}}{2 G \Sigma_{g=1}^{G} \Sigma_{u=1}^{2}\left(R_{g, u}\right)^{2}}
$$

$F_{p}$ is a real number in the interval $\left[\frac{1}{2 G}, 1\right]$ with the maximum value of one when equal data rate is achieved for all the users, and minimum value of $\frac{1}{2 G}$ when one user dominates the whole bandwidth and takes over all the subcarriers.

\section{SIMULATION RESULTS}

This section provides the simulation results obtained for the proposed LP based algorithms. The proposed algorithm has been simulated in MATLAB. The performances of the proposed algorithm has also been compared with the existing algorithm given in [8]. The simulation parameters have been given in Table-1.

Table-1 Simulation parameters

\begin{tabular}{|l|l|}
\hline Total available bandwidth, $\mathrm{B}$ & $1 \mathrm{MHz}$ \\
\hline Total transmit power, $\mathrm{P}$ & $1 \mathrm{~W}$ \\
\hline Number of subcarriers, $\mathrm{N}$ & 256 \\
\hline Number of users per group, $\mathrm{U}$ & 2 \\
\hline Single sided Noise power spectral density, $\mathrm{N}_{0}$ & $10^{-8} \mathrm{~W} / \mathrm{Hz}$ \\
\hline Cooperation coefficient, $\psi$ & 0.9 \\
\hline
\end{tabular}

The channel model is employed as frequency selective with independent multipath and exponential profile. A total of 256 subcarriers have been considered for allocations to the users in the downlink scenario, so that all subcarriers experience flat fading. This number $(\mathrm{N}=256)$ is also large enough for the duality gap to vanish. It is assumed that full channel state information (CSI), including an inter user channel, is available at the base station. Equal cooperation coefficient, $\psi$ is assumed among the users of different groups. For comparison purpose and ease of analysis, the value chosen for cooperation coefficient, $\psi$ is 0.9 . The results are averaged based on 100 channel realizations.

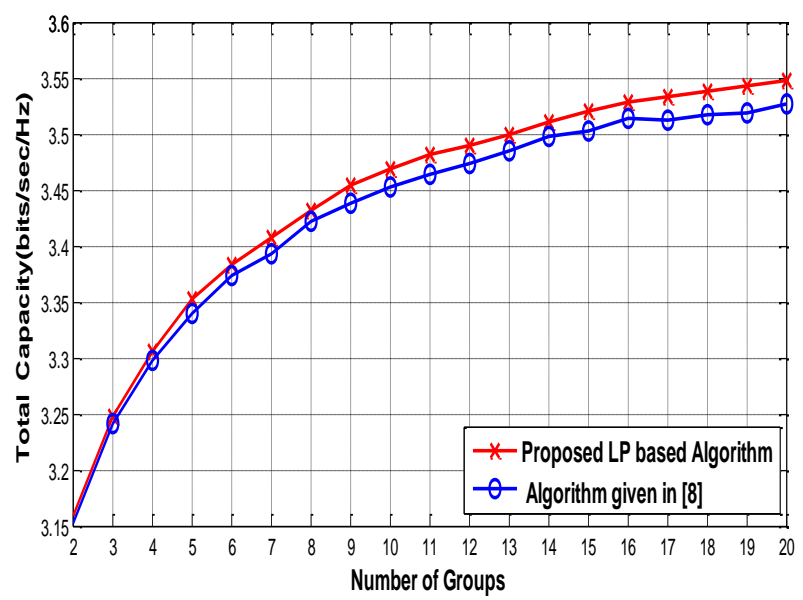

Fig. 2. Throughput versus number of groups for $\psi=0.9$

Fig 2 shows the variation in achievable total capacity in the system with the number of groups. As the number of groups

increases, the total capacity increases due to increase in the number of users. This figure also shows the comparison of proposed algorithm with the algorithm given in [8]. It can be seen that the achievable total capacity in the system using proposed algorithm is higher than that achievable using the algorithm given in [8]. 


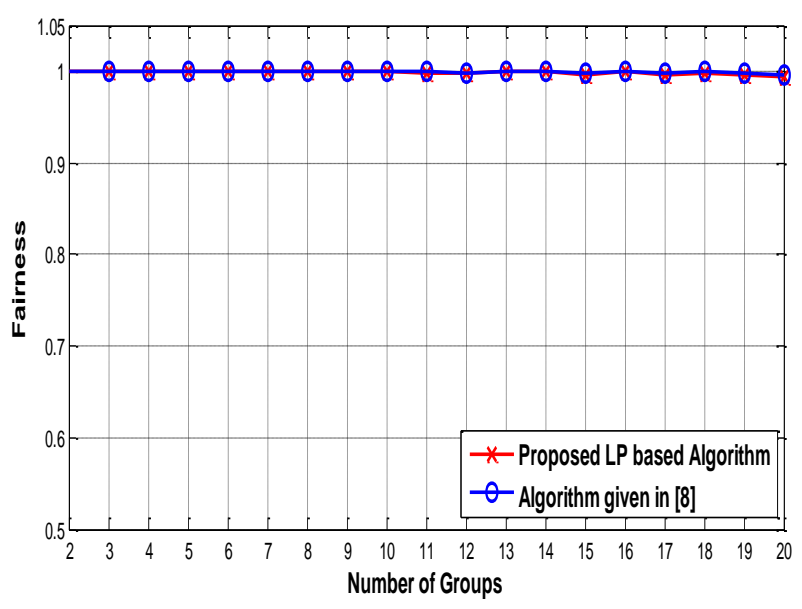

Fig. 3. Fairness versus number of groups for $\psi=0.9$

Fig. 3 presents the plot of fairness index, $F_{p}$, vs number of groups in the system. As it can be seen that the achievable fairness index in proposed algorithm and algorithm given in [8] is almost equal and maintain fairness index close to one, which means that there is no loss of fairness in the system for increasing the total capacity of the system by using proposed LP based subcarrier allocation algorithm.

\section{CONCLUSION}

An efficient LP based subcarrier allocation algorithm for downlink of an OFDMA cooperative system with grouped users is proposed in this paper. The proposed algorithm transforms the non-convex optimization problem into a convex optimization problem and utilizes the MATLAB tool "linprog" to solve this convex optimization problem. The proposed algorithm allocates subcarriers efficiently among users so that the total capacity in the system is maximized. The proposed LP based subcarrier allocation algorithm also ensures the fairness among the users by keeping equal data rate of all users. The performance of our LP based subcarrier allocation algorithm in terms of total system capacity has been compared with an existing algorithm given in [8] and results show that higher total system capacity can be achieved by the proposed LP based subcarrier allocation algorithm while maintaining fairness among users in the system.

\section{REFERENCES}

[1] Y. Li and G. L. St uber, Orthogonal Frequency Division Multiplexing for Wireless Communications. Springer, 2006.

[2] A. Nosratinia, T.E Hunter, A, Hedayat, "Cooperative communication in wireless networks", IEEE Communications Magazine, vol. 42, pp. 74-80, Oct. 2004.

[3] J. Jang and K. B. Lee, "Transmit power adaptation for multiuser OFDM systems," IEEE J. Sel. Areas Commun., vol. 21, pp. 171-178, Feb. 2003

[4] Z. Han, T. Himsoon, W. P. Siriwongpairat, and K. J. Ray Liu, "Resource allocation for multiuser cooperative OFDM networks: Who helps whom and how to cooperate," IEEE Trans. Veh. Technol., vol. 58, pp. 2378-2391, Jun. 2009.

[5] A. Nosratinia and T. E. Hunter, "Grouping and partner selection in cooperative wireless networks," IEEE J. Sel. Areas Commun., vol. 25, pp. 369-378, Feb. 2007.

[6] J. N. Laneman, D. N. C. Tse, and G.W.Wornell, "Cooperative diversity in wireless networks: Efficient protocols and outage behavior," IEEE Trans.Inf. Theory, vol. 50, pp. 3062-3080, Dec. 2004.

[7] R. Nabar, H. Bolcskei, and F. Kneubuhler, "Fading relay channels: Performance limits and space-time signal design," IEEE Journal on Selected Areas in communications, vol. 22, pp. 1099-1109, Aug. 2004.

[8] H. Rasouli, S. Sadr and A. Anpalagan, "A fair subcarrier allocation algorithm for cooperative wireless networks with grouped users", IEEE Global Communication Conference, pp. 1-6, December 2008.

[9] W. Yu and R. Lui, "Dual methods for nonconvex spectrum optimization of multicarrier systems", IEEE Trans. Commun. vol. 54, pp. 1310-1322 July 2006.

[10] Zhang, Y., Solving Large-Scale Linear Programs by Interior-Point Methods Under the MATLAB Environment, Technical Report TR96-01, Department of Mathematics and Statistics, University of Maryland, Baltimore County, Baltimore, MD, July 1995.

[11] S. Mehrotra, On the Implementation of a Primal-Dual Interior Point Method, SIAM Journal on Optimization, Vol. 2, pp. 575-601, 1992. 